\title{
Oral Antibiotics in Clinical Development for Community-Acquired Urinary Tract Infections
}

Balaji Veeraraghavan · Yamuna Devi Bakthavatchalam •

Rani Diana Sahni

Received: May 16, 2021 / Accepted: July 21, 2021 / Published online: August 6, 2021

(C) The Author(s) 2021

\section{ABSTRACT}

The treatment of urinary tract infections (UTIs) has been complicated by the emergence of multidrug-resistant, $\quad \beta$-lactamase-expressing pathogens. As a result of the limited treatment options, patients often require hospitalization and intravenous therapy. In essence, a strong unmet need for oral antibiotics, active against extended-spectrum $\beta$-lactamase (ESBL) uropathogens has emerged. Oral carbapenems (tebipenem and sulopenem) and oral cephalosporin/ $\beta$-lactamase inhibitor combinations are in various stages of clinical development for the treatment of uncomplicated and complicated UTI. Tebipenem, if approved, will be the first oral treatment for complicated UTI while sulopenem will be for uncomplicated
UTI. The $\beta$-lactamase inhibitors ETX0282, VNRX7145, ARX1796, and QPX7728 are combined with cefpodoxime proxetil or ceftibuten that achieve favorable exposures in urine compared to other uropathogen-active oral cephalosporins. The combination ceftibutenQPX7728 has potential broad-spectrum coverage against carbapenemase producers including metallo $\beta$-lactamase producers. Other novel combinations, namely cefpodoxime/ETX0282, ceftibuten/VNRX-7145, and ceftibuten/ ARX1796, have also demonstrated excellent activity against Klebsiella pneumoniae carbapanemase (KPC) and OXA-48-like producers. All these agents, upon their arrival for commercial use, would strengthen the outpatient therapy.

Keywords: Ceftibuten;

Cefpodoxime; Tebipenem; Sulopenem; ETX0282; VNRX7145; ARX1796; QPX7728

B. Veeraraghavan $(\bowtie) \cdot$ Y. D. Bakthavatchalam .

R. D. Sahni

Department of Clinical Microbiology, Christian

Medical College, Vellore, Tamil Nadu 632004, India

e-mail: vbalaji@cmcvellore.ac.in 


\section{Key Summary Points}

The treatment of urinary tract infections (UTIs) has been complicated by the emergence of multidrug-resistant and extended-spectrum $\beta$-lactamase (ESBL)expressing Gram-negative pathogens.

Oral carbapenems (tebipenem and sulopenem) and oral cephalosporin/ $\beta$ lactamase inhibitor combinations are in various stages of clinical development for treating UTIs.

Recently tebipenem and sulopenem have completed phase III trials. Tebipenem, if approved, will be the first oral treatment for complicated UTI while sulopenem will be for uncomplicated UTI.

The combinations cefpodoxime/ETX0282, ceftibuten/VNRX-7145, and ceftibuten/ QPX7728 are in phase I development. All these agents, upon their arrival for commercial use, would strengthen the outpatient therapy.

\section{INTRODUCTION}

Pharmaceutical organizations that are in antibiotic discovery research prioritize their projects on the basis of evolving antibiotic resistance epidemiology. For instance, the emergence and spread of methicillin-resistant Staphylococcus aureus, vancomycin-resistant Enterococcus faecium, and macrolide-resistant Streptococcus pneumoniae during 1980s and 1990s compelled these organizations to focus on discovery of novel agents to tackle these Gram-positive pathogens. Emphasis on Gramnegative pathogens that we witness today started when carbapenem-resistant Klebsiella pneumoniae through expression of a serine carbapenemase (KPC) was reported in New York during the 2000s [1]. Needless to say, extendedspectrum $\beta$-lactamases (ESBLs) and class $C \beta$ - lactamases in Enterobacterales were a problem even before, but the former are addressed by piperacillin/tazobactam to some extent and carbapenems while the latter are addressed by carbapenems alone [2].

In the last decade, we experienced global spread of KPCs and also other carbapenemases, viz., New Delhi metallo $\beta$-lactamases and OXA$\beta$-lactamases [3]. What we have failed to notice is the quiet increase in ESBL pathogens in community-onset urinary tract infection (UTI), a condition usually managed by oral antibiotics such as nitrofurantoin, trimethoprim/sulfamethaxozole, quinolones, and oral cephalosporins. The fact that these uropathogens carrying ESBLs (thus resistant to oral cephalosporins) have acquired resistance to the aforementioned non- $\beta$-lactam antibiotics (Table 1 ) is challenging for treatment [31]. As a result, in today's scenario, in the USA alone, huge numbers of patients with UTIs are hospitalized because of the failure of oral antibiotics and left with intravenous antibiotic options [32, 33]. In these cases, even piperacillin/tazobactam is often not active because of widely prevalent class $\mathrm{C}$ and OXA-1 $\beta$-lactamases, thus forcing carbapenem use [2]. In essence, a strong unmet need for oral antibiotics that are active against ESBL uropathogens has emerged. Moreover, with an additional activity against carbapenemresistant uropathogens, these oral drugs can be used as step-down for hospital-treated patients with UTIs, enabling early hospital discharge. In this review, we describe the profile and status of novel oral antibiotics in clinical development. This article is based on previously conducted studies and does not contain any studies with human participants or animals performed by any of the authors.

\section{CARBAPENEMS}

Historically, discovering an orally bioavailable carbapenem remained a challenge to medicinal chemists. Initially, the aim was to develop a carbapenem for outpatient treatment of community-origin lower respiratory infections. With this thinking, faropenem was developed and approved in Japan. In the USA, a prodrug of 







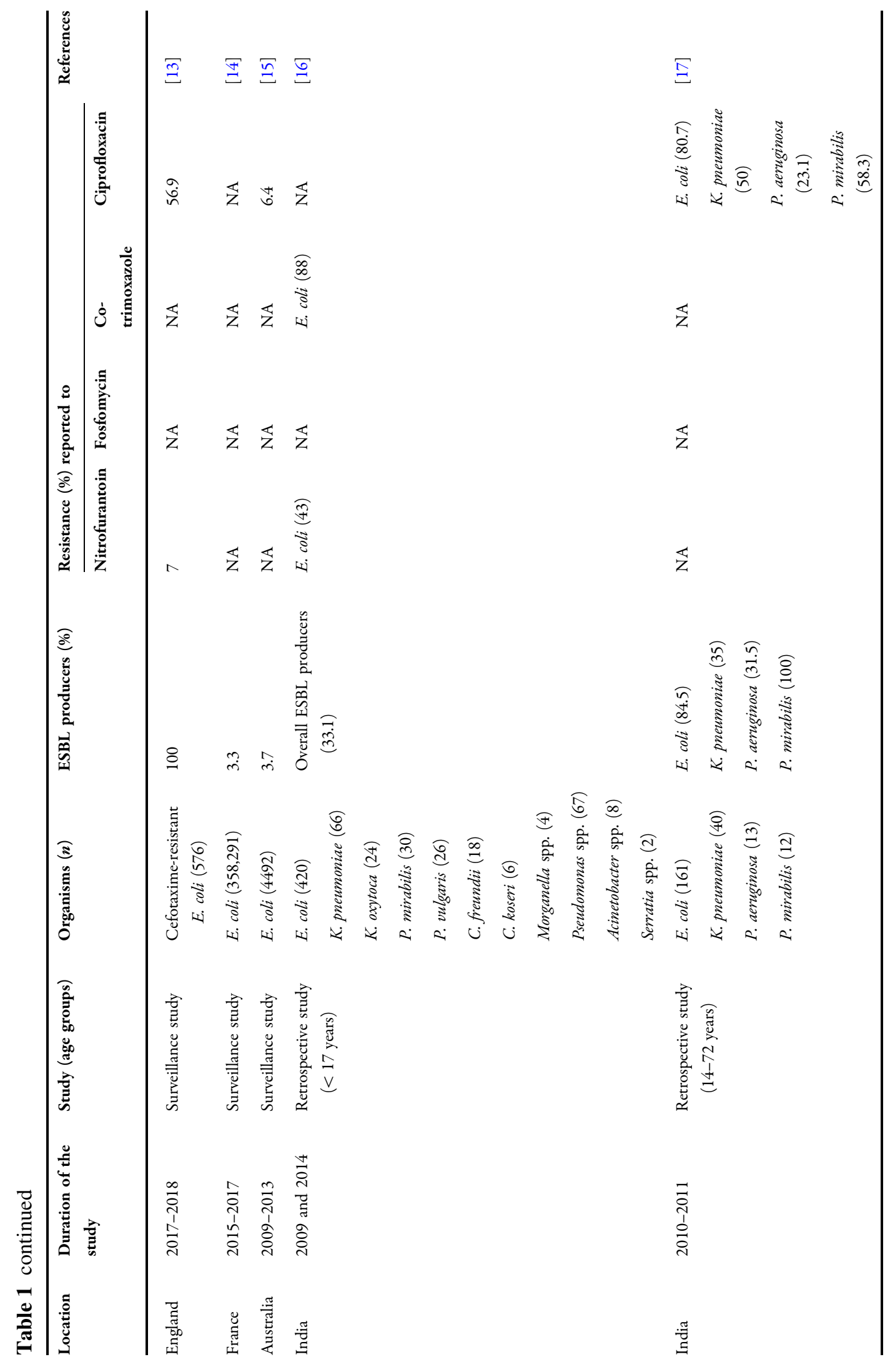









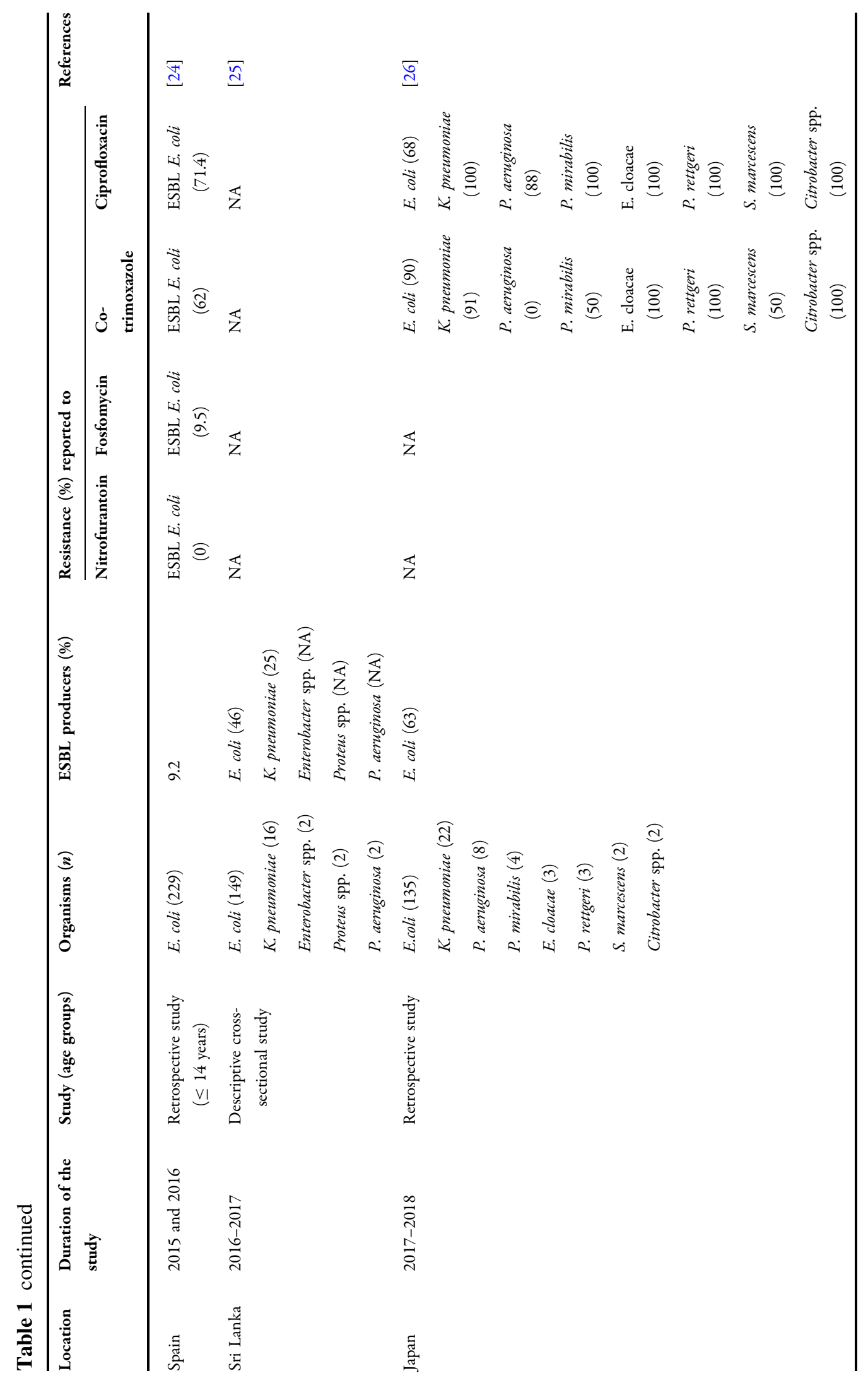




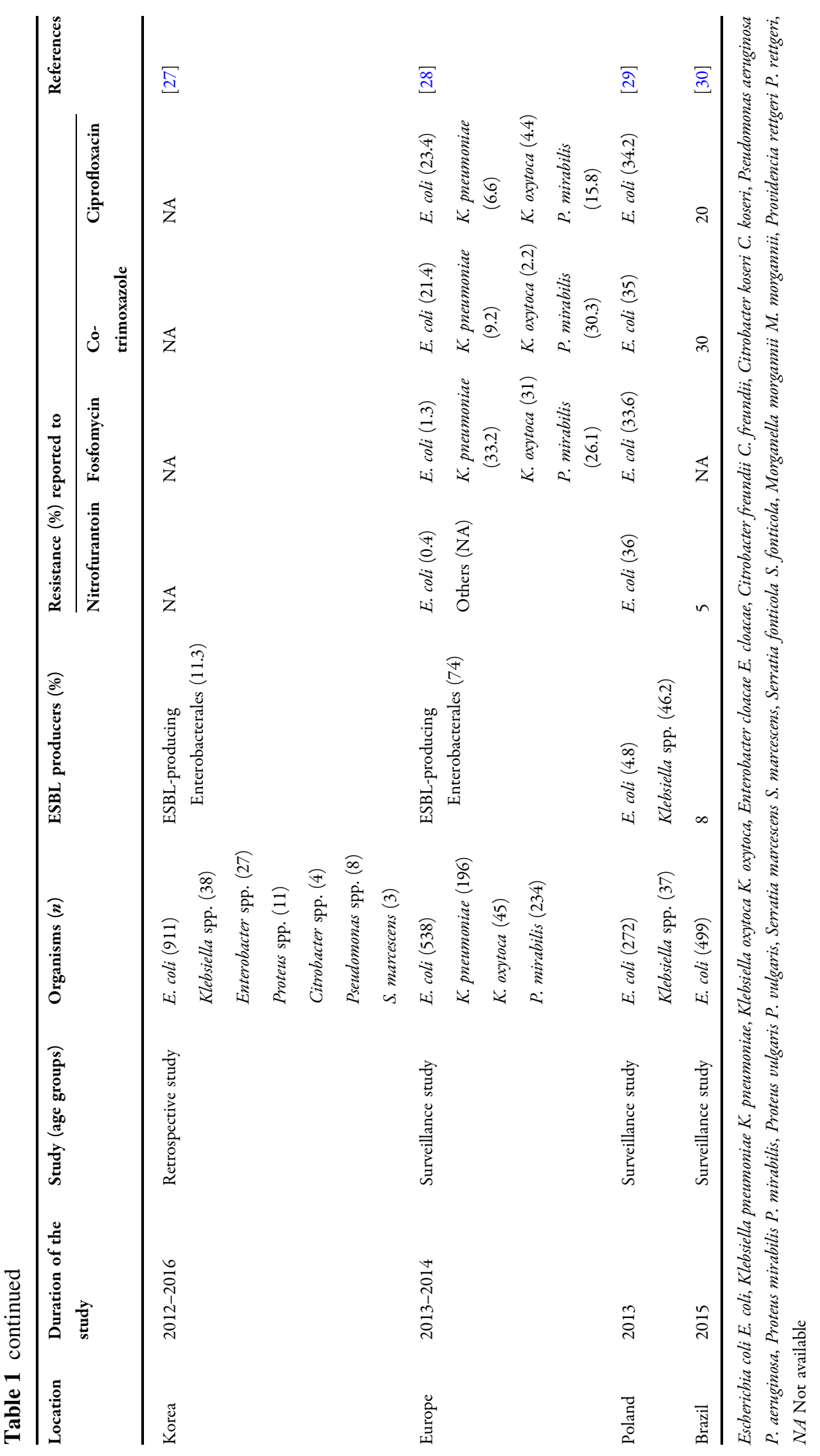


Table 2 Spectrum of activity of oral antibiotics against Gram-negative pathogens causing community-acquired urinary tract infections

\begin{tabular}{|c|c|c|c|c|c|}
\hline \multirow[t]{3}{*}{ Oral antibiotics } & \multicolumn{5}{|c|}{ Activity spectrum } \\
\hline & \multirow[t]{2}{*}{ ESBLs } & \multirow[t]{2}{*}{ ampC } & \multicolumn{3}{|l|}{ CRE } \\
\hline & & & $\overline{\mathrm{KPC}}$ & MBL & OXA-48-like \\
\hline Tebipenem pivoxil hydrobromide & $\checkmark$ & $\checkmark$ & $\mathrm{X}$ & $\mathrm{X}$ & $\mathrm{X}$ \\
\hline Sulopenem-etzadroxil/probenecid & $\boldsymbol{v}$ & $\boldsymbol{v}$ & $\mathrm{X}$ & $\mathrm{X}$ & $\mathrm{X}$ \\
\hline Cefpodoxime/ETX0282 & $\checkmark$ & $\checkmark$ & $\checkmark$ & $\mathrm{X}$ & $\checkmark$ \\
\hline Ceftibuten/VNRX-7145 & $\boldsymbol{v}$ & $\checkmark$ & $\checkmark$ & $\mathrm{X}$ & $\checkmark$ \\
\hline Ceftibuten/ARX1796 & $\checkmark$ & $\checkmark$ & $\checkmark$ & $\mathrm{X}$ & $\boldsymbol{V}$ \\
\hline Ceftibuten/ QPX7728 & $\checkmark$ & $\checkmark$ & $\checkmark$ & $\checkmark$ & $\checkmark$ \\
\hline
\end{tabular}

$\checkmark$ active, $X$ not active, ESBL extended-spectrum $\beta$-lactamases, ampC class $C$ cephalosporinase, KPC K. pneumoniae carbapanemases, $M B L$ metallo $\beta$-lactamases, OXA-48 oxacillinase, CRE carbapenem-resistant Enterobacterales, CRPA carbapenem-resistant $P$. aeruginosa, $C R A B$ carbapenem-resistant $A$. baumannii

faropenem was developed but ran into regulatory hurdles and was never approved by the US Food and Drug Administration (FDA) [34]. Subsequently, interest in this area waned but returned as resistance to first-line oral antibiotics has increased to a substantial level among community Gram-negative infections. The spectrum of activity of oral carbapenems is given in Table 2 . The pharmacokinetic characteristics and in vitro activity of oral carbapenems against urinary isolates of ESBL-producing Enterobacterales are given in Table 3. Two oral carbapenems, tebipenem and sulopenem, have been evaluated in phase 3 studies for the treatment of UTIs (Table 4).

\section{Tebipenem}

Tebipenem pivoxil (TBPM-PI) an orally administered prodrug of tebipenem, marketed in Japan by Meiji Seika Pharma Co., Ltd for the treatment of otitis media, sinusitis, and pneumonia in pediatric patients [44]. This oral formulation was not marketed for adult patients. A new formulation for adults consisting of tebipenem pivoxil hydrobromide (TBPM-PI-HBr) salt was designed by Spero Therapeutics to improve the drug substance and drug product properties such as stability [45]. TBPM-PI-HBr is being developed by Spero Therapeutics for the treatment of complicated UTI (cUTI) including acute pyelonephritis (AP). The drug is being promoted as potential treatment option for cUTI caused by Enterobacterales resistant to first-line oral agents, nitrofurantion, sulfamethaxazole/trimethoprim, fluoroquinlones, and oral cephalosporins. It is not active against carbapenem-resistant Enterobacterales.

Tebipenem is highly hydrophilic, which significantly limits its oral absorption. In the past, prodrug strategies have been employed to improve the oral bioavailability of many $\beta$-lactam antibiotics. The prodrug TBPM-PI-HBr rapidly gets converted to active tebipenem in the plasma and enterocytes [46]. The pharmacokinetic analysis of single and multiple ascending oral doses of TBPM-PI-HBr indicates that a dose regimen $600 \mathrm{mg} \mathrm{q} 8 \mathrm{~h}$ provides bioavailability in the range of $50-60 \%$ [47]. However, the protein binding of tebipenem $(98.7 \%)$ is likely higher than ertapenem (90-95\%) [48]. Approximately $55-60 \%$ of the total tebipenem dose is recovered in the urine as an intact drug, which is 50-100-fold greater than that of free plasma tebipenem concentration [47]. Consequently, such a high urine exposure makes it suitable for the treatment of UTIs. 


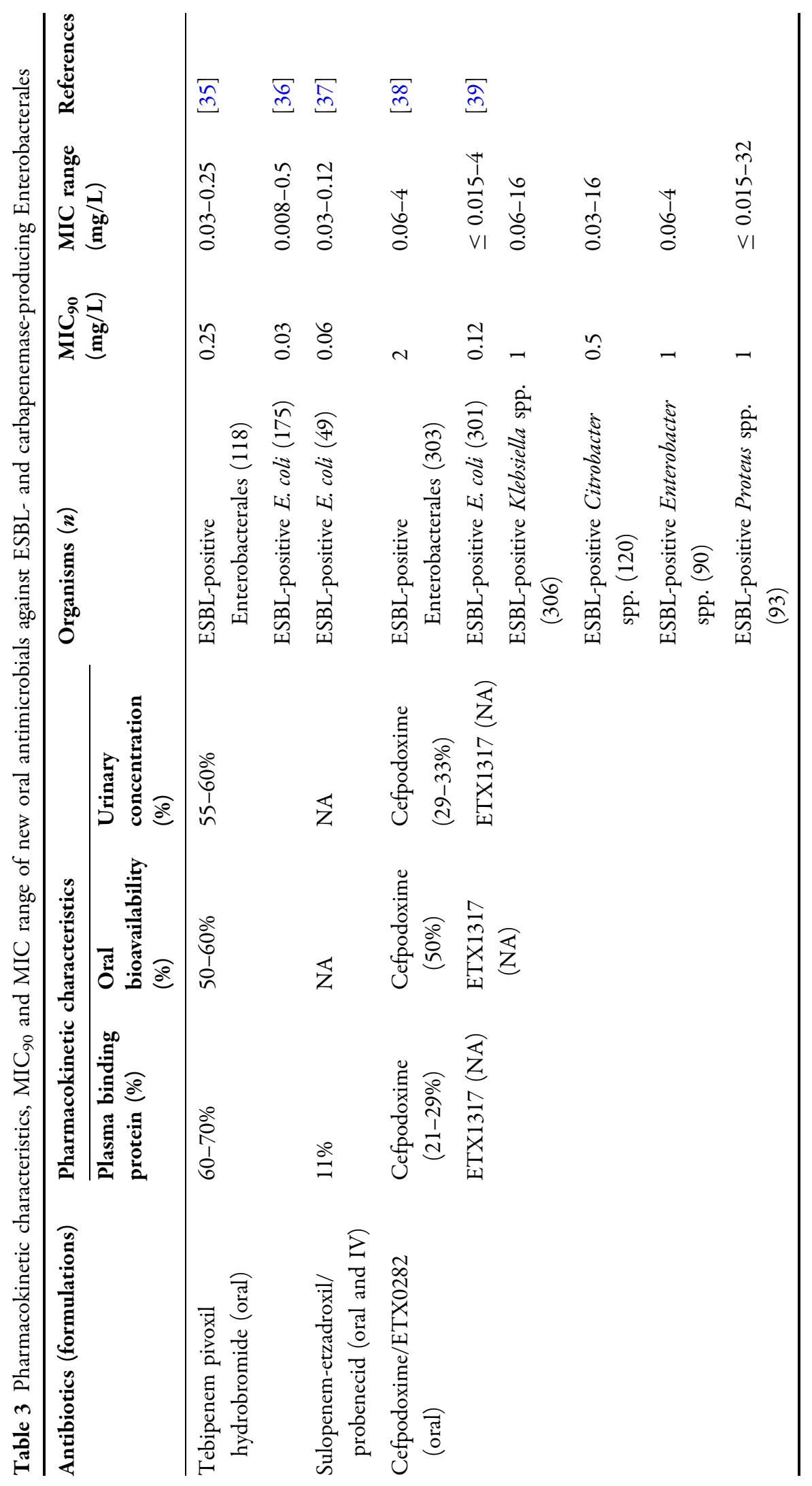




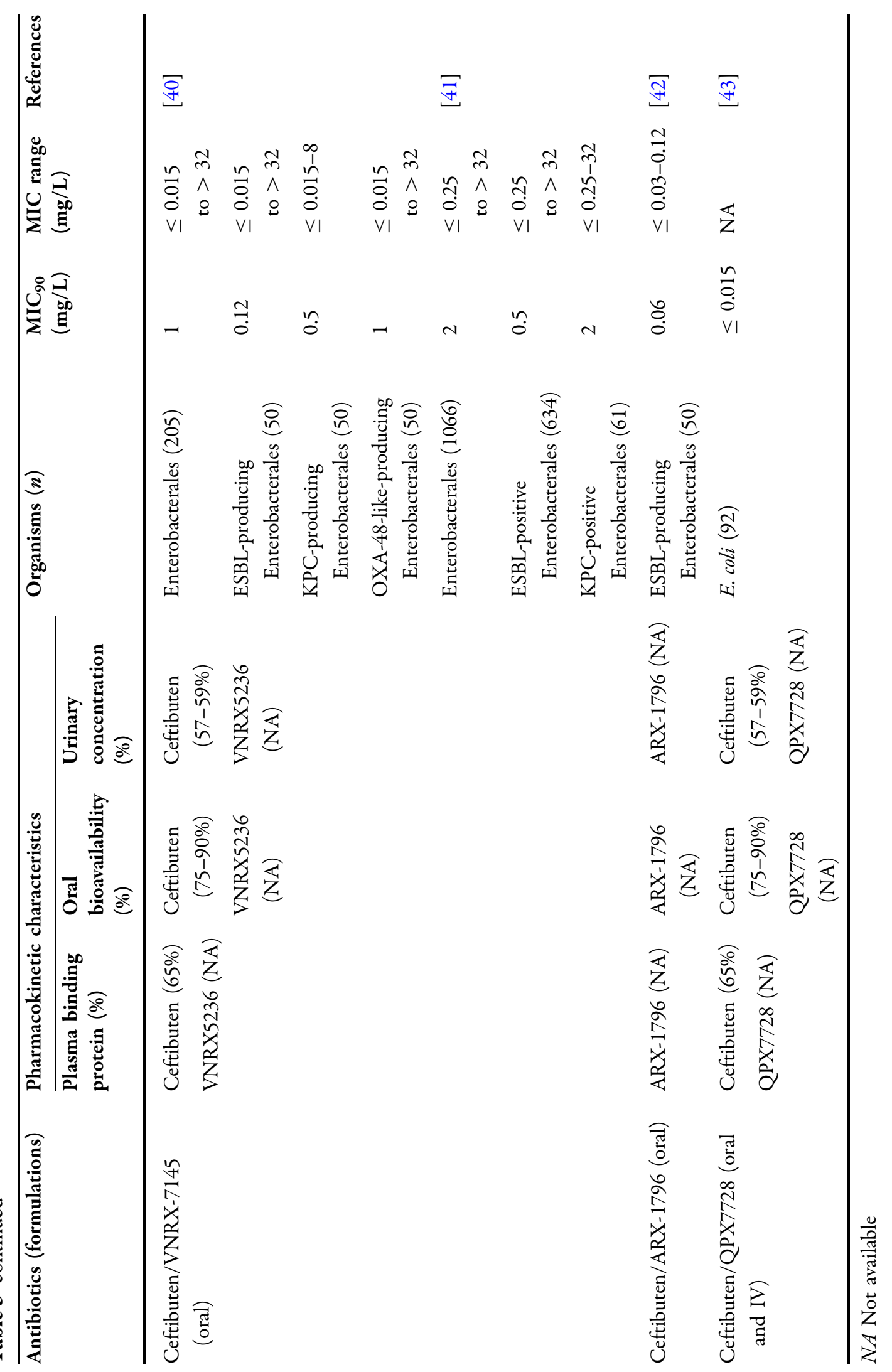









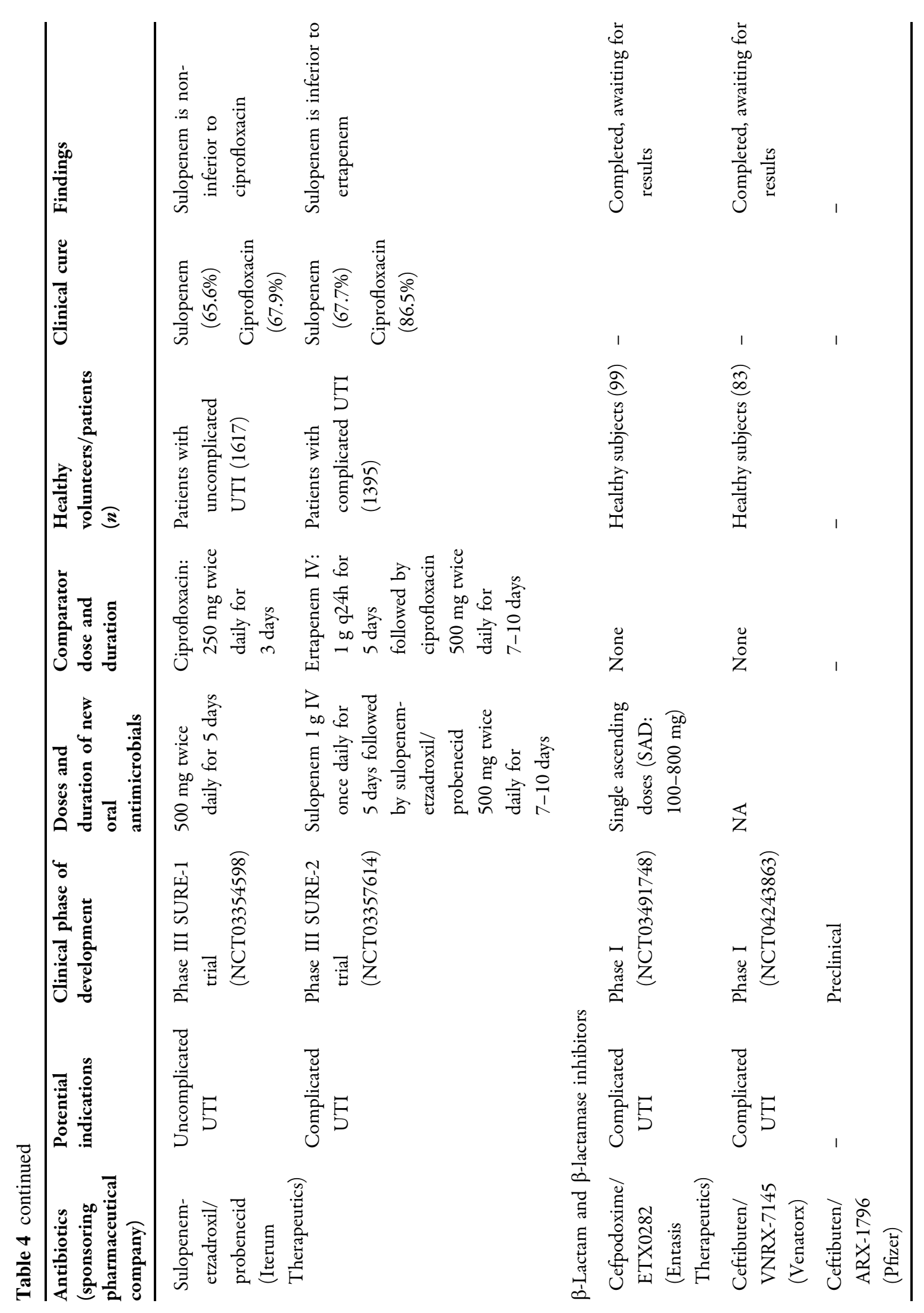







Unsurprisingly, it is not stable to carbapenemases. Iterum undertook three phase 3 studies for sulopenem (Table 4). Sulopenem etzadroxil $500 \mathrm{mg} /$ probenecid $500 \mathrm{mg}$ is the first antibiotic evaluated for the indication of uncomplicated UTI [53]. It should be noted that until recently, there has been no FDA guideline for the conduct of phase 3 study for the indication of uncomplicated UTI. In the all-oral therapy study (SURE-1), sulopenem etzadroxil $500 \mathrm{mg} /$ probenecid $500 \mathrm{mg}$, q12h for 5 days demonstrated non-inferiority to ciprofloxacin $250 \mathrm{mg}$, $\mathrm{q} 12 \mathrm{~h}$ for 3 days [54]. However, in the phase 3 studies for the indication of cUTI (SURE-2) and complicated intra-abdominal infections (SURE3), a 5-day once-daily $1 \mathrm{~g}, 2$-h infusion, IV sulopenem followed by orally administered sulopenem etzadroxil $500 \mathrm{mg} /$ probenecid $500 \mathrm{mg}, \mathrm{q} 12 \mathrm{~h}$ step-down failed to meet the non-inferiority margin for the primary endpoint [55]. In both studies, the comparator was ertapenem $1 \mathrm{~g}$, q24h, IV for 5 days followed by an oral step-down therapy.

Iterum submitted the new drug application to the FDA in December 2020 for the approval of sulopenem oral prodrug formulation for the indication of uncomplicated UTI.

\section{NOVEL B-LACTAM AND B- LACTAMASE INHIBITORS}

The challenge in discovering orally bioavailable $\beta$-lactams is evident from the reality that there is only a single oral $\beta$-lactamase inhibitor, clavulanic acid, in clinical use [56]. The combination of amoxicillin and clavulanic acid is generally not preferred for UTI for two reasons: (1) the concentration of intact clavulanic acid eliminated in the urine is low and (2) clavulanic acid is not an inhibitor of class $C$ (and also an inducer) and OXA-1 enzymes commonly associated with ESBL uropathogens [57]. Combining clavulanic acid with an oral cephalosporin was attempted (ceftibuten + clavulanic acid) which is certainly a better combination than amoxicillin + clavulanic acid [58]. Entasis seems to be the first company that ventured into discovering a prodrug of diazabicyclooctane with good oral bioavailability. A few more companies joined the race and one objective that is common among them is to develop an oral $\beta$-lactamase inhibitor with an expanded spectrum of activity against ESBLs, class $\mathrm{C}$, and carbapenemases (Tables 2 and 3). These $\beta$-lactamase inhibitors are combined with cefpodoxime proxetil or ceftibuten (Table 4), the two oral cephalosporins that achieve favorable exposures in urine compared to other Gram-negative uropathogens-active oral cephalosporins, cefuroxime axetil and cefixime. All these oral $\beta$ lactam and $\beta$-lactamase inhibitors are undergoing phase I clinical trials (Table 4), except ceftibuten/ARX-1796 which is at the preclinical stage of development.

\section{Cefpodoxime/ETX0282}

Cefpodoxime proxetil is an orally administered prodrug which is absorbed and de-esterified by the intestinal mucosa to release the active metabolite cefpodoxime with $50 \%$ systemic bioavailability [59]. Approximately $29-33 \%$ of the administered cefpodoxime dose is eliminated unchanged by renal excretion, which makes it as a suitable drug for treating UTIs [60]. Cefpodoxime proxetil has been approved by FDA for multiple indications that include community-origin upper and lower respiratory infections, uncomplicated skin and skin structure infections, and uncomplicated UTI (cystitis). The highest recommended total daily dose is $800 \mathrm{mg}$ of active cefpodoxime ( $400 \mathrm{mg}$, $\mathrm{q} 12 \mathrm{~h}$ ) [60].

ETX0282 is a prodrug of ETX1317, a novel diazabicyclooctane-based inhibitor of class A, C, and $\mathrm{D} \beta$-lactamases [61]. Thus the combination ETX1317 and cefpodoxime is active against ESBL/class C/KPC-expressing Enterobacterales excluding metallo $\beta$-lactamases (Table 3). ETX1317 also has some PBP2 binding and therefore shows standalone activity mainly against E. coli with MICs in the range of 0.5 to $1 \mathrm{mg} / \mathrm{L}$ [62]. Owing to this standalone activity, the MIC of cefpodoxime/ETX 1317 is determined at 1:2 ratio, rather than using a fixed $4 \mathrm{mg} / \mathrm{L}$ as in the case of other $\beta$-lactam and $\beta$ lactamase inhibitor combinations [35]. The originator is Entasis Therapeutics Ltd., 
(Massachusetts, USA) and presently the combination is in phase 1 development.

The current susceptible breakpoint of cefpodoxime for Enterobacterales is $2 \mathrm{mg} / \mathrm{L}$. In a global surveillance study, against 1875 Enterobacterales isolated from patients with UTIs, cefpodoxime/ETX 1317 showed $\mathrm{MIC}_{50 / 90}$ of 0.06/0.12 mg/L [36]. Against ESBL- and ampCproducing Enterobacterales subsets, $\mathrm{MIC}_{50 / 90}$ values of this combination were $0.12 / 0.25$ and $0.25 / 1 \mathrm{mg} / \mathrm{L}$, respectively [35]. Thus, in sum, the MIC $_{90}$ of cefpodoxime/ETX1317 remained below the current cefpodoxime breakpoint of $2 \mathrm{mg} / \mathrm{L}$ for class $\mathrm{A}, \mathrm{C}$, and D-expressing $\beta$ lactamases.

The preliminary phase I results (Table 4) showed rapid absorption of ETX0282 tested at various doses (100-800 $\mathrm{mg}$ ), plasma concentrations were reported to be in the expected therapeutic range, and there was no drug-drug interaction between ETX0282 and cefpodoxime proxetil [63]. Generally, ETX0282 is well tolerated either alone or in combination with cefpodoxime proxetil, with no serious adverse events, though mild to moderate emesis has been documented. Additional studies are warranted to further investigate the potential correlation between absorption profile and emesis and to formulate ETX0282 for further clinical development. The bioavailability of ETX0282 observed from the phase 1 studies has not been reported. In preclinical species (rat, dog, and monkey) it showed at least $78 \%$ bioavailability [64].

If cefpodoxime proxetil/ETX0282 is developed successfully, we think its main usage would be for the outpatient management of patients with UTIs and suspected or confirmed ESBL pathogen who do not respond to first-line oral agents. The reason is extremely low prevalence of CREs in the community setting.

\section{Ceftibuten/VNRX-7145}

Ceftibuten was originally approved by the FDA for the treatment of acute exacerbations of chronic bronchitis, acute otitis media, and pharyngitis caused by Haemophilus influenzae, Moraxella catarrhalis, or Streptococcus pneumoniae in 1995. However, it is not approved in European countries. Ceftibuten is chosen off-label for the treatment of UTI; however, no dosing recommendations exist. Ceftibuten is rapidly absorbed (75-90\%) from the gastrointestinal tract in its active form following oral administration, of which $65 \%$ binds to plasma proteins [65]. It has a half-life of $2.5 \mathrm{~h}$ and eliminated mainly in urine (57-59\%) and feces (39\%) [66]. Ceftibuten has an excellent systemic exposure following oral doses and adequately achieves its pharmacokinetics (PK) target.

VNRX-7145 is a novel cyclic boronate $\beta$-lactamase inhibitor with potent inhibitory activity against class A, C, and D $\beta$-lactamases [67]. In vivo, VNRX-7145 undergoes biotransformation to an active inhibitor, VNRX-5236 [68]. It is being developed in combination with ceftibuten as an oral therapy by VenatoRx Pharmaceuticals, Inc. (Pennsylvania, USA). The combination of VNRX-5236 with ceftibuten was tested against a collection of 205 Enterobacterales isolates and showed a $\mathrm{MIC}_{50 / 90}$ of 0.12 / $1 \mathrm{mg} / \mathrm{L}$ [37]. This combination also retained its potent activity against $\mathrm{KPC}\left(\mathrm{MIC}_{50 / 90}, 0.12 /\right.$ $0.5 \mathrm{mg} / \mathrm{L})$ and OXA-48-like producers $\left(\mathrm{MIC}_{50 / 90}\right.$, $0.25 / 1 \mathrm{mg} / \mathrm{L}$ ) [37]. This MIC data suggests that addition of VNRX-5236 maintains twofold lower MICs, compared to ceftibuten susceptibility breakpoint $(\leq 2 \mathrm{mg} / \mathrm{L})$.

In combination with ceftibuten, the fAUC $_{0-24} / \mathrm{MIC}$ is the $\mathrm{PK} /$ pharmacodynamics (PD) index that appeared to drive in vivo efficacy of VNRX-5236 [69]. Furthermore, addition of VNRX-7145 to ceftibuten significantly reduced bacterial burden in a murine UTI model using ESBL- and KPC-2-producing E. coli and in a murine thigh infection model using OXA-48and KPC-producing Enterobacterales. A phase I trial (NCT04243863) is currently enrolled to assess the safety and PK of VNRX-7145 using single and multiple ascending doses (Table 4).

Since VNRX-7145 is not an inhibitor of MBL, the anticipated therapeutic spectrum of ceftibuten/VNRX-7145 combination is comparable to that of cefpodoxime/ETX0282. 


\section{Ceftibuten/ARX1796}

Avibactam is a potent DBO inhibitor of class A, $\mathrm{C}$, and some D $\beta$-lactamases approved for IV dosing in combination with ceftazidime by the FDA in 2015, but the oral bioavailability of avibactam is negligible (ca. 7\%) [70]. To expand the clinical utility of avibactam, Arixa Pharmaceuticals (Palo Alto, CA, USA) has discovered a novel orally administered avibactam prodrug (ARX-1796/AV-006) [71]. The prodrug ARX1796 has been shown to have an oral bioavailability of $60-80 \%$ in phase 1 studies [72]. The oral $\beta$-lactams ceftibuten, cefixime, amoxicillin, cefpodoxime, sulopenem, and tebipenem were evaluated in combination with ARX-1796 against Enterobacterales expressing ESBL, ampC, KPC, and OXA-48-like [73]. Ceftibuten with ARX-1796 showed lower MICs compared to other combinations. Addition of ARX-1796 to ceftibuten retained its potent activity against KPC and OXA-48-like producers with the low $\mathrm{MIC}_{50 / 90}$ of $0.06 / 0.5 \mathrm{mg} / \mathrm{L}$ and $0.06 / 0.25 \mathrm{mg} / \mathrm{L}$, respectively [39]. Interestingly, pharma giant Pfizer took over Arixa Pharmaceuticals recently.

\section{Ceftibuten/QPX7728}

QPX7728 is an ultrabroad-spectrum boronic acid $\beta$-lactamase inhibitor being developed by Qpex Biopharma (San Diego, USA) [74]. Ceftibuten with QPX7728 and meropenem with QPX7728 are in clinical development as an oral and intravenous formulation, respectively [75]. Unlike other orally bioavailable $\beta$-lactamase inhibitors (ETX0282, VNRX1745, ARX1796), QPX7728 inhibits serine and metallo $\beta$-lactamases of classes A, B, C, and D in Enterobacterales, Pseudomonas aeruginosa, and Acinetobacter spp.

Tebipenem and cefibuten were evaluated in combination with QPX7728 against Enterobacterales with varying resistance mechanisms. Tebipenem-QPX7728 $\quad\left(\mathrm{MIC}_{50 / 90}, \leq 0.06 / 2 \mathrm{mg} /\right.$ $\mathrm{L})$ is more potent than ceftibuten-QPX7728 combination $\left(\mathrm{MIC}_{50 / 90}, 0.25 / 16 \mathrm{mg} / \mathrm{L}\right)$; however, impermeability decreases the potency of tebipenem-QPX7728 [76].
In preclinical species, QPX7728 has oral bioavailability of $43-53 \%$; however, saturation is likely to be expected with higher doses. QPX7728 is about $89 \%$ plasma protein-bound [77]. Approximately 64\% of the QPX7728 dose was recovered unchanged in the urine [78]. A phase I study (NCT04380207) is currently underway to assess the safety and PK of QPX7728 alone following single and multiple intravenous doses, and in combination with a $\beta$-lactam antibiotic.

\section{CONCLUSION}

The ongoing pandemic reiterates the need for best use of hospital resources and to achieve this target, it is necessary to minimize avoidable hospitalization. Since the time Gram-negative pathogens emerged as problematic pathogens because of antibiotic resistance, the focus remained mainly on hospital-treated patients. Meanwhile, first-line oral antibiotics used to treat infections caused by community-onset Gram-negatives, especially the uropathogens, are increasingly non-effective leading to increasing rates of hospitalization. Therefore, there is an unmet need for oral antibiotics active against antibiotic-resistant Gram-negative pathogens encountered in community infections, particularly in UTI. Being safe and having the tendency to achieve higher urinary concentrations, $\beta$-lactams have become the preferred choice. As the main challenge to $\beta$ lactams is the $\beta$-lactamases, novel $\beta$-lactamase inhibitors with expanded inhibitory spectrum and good oral bioavailability were discovered and combined with already approved $\beta$-lactams. Tebipenem, if approved, will be the first oral treatment for cUTI while sulopenem will be for uncomplicated UTI. Both lack activity against CREs, and CRE-active oral agents are in phase 1 development. All these agents, upon their arrival for commercial use, would strengthen the outpatient therapy. 


\section{ACKNOWLEDGEMENTS}

Funding. No funding or sponsorship was received for this study or publication of this article.

Authorship. All named authors meet the International Committee of Medical Journal Editors (ICMJE) criteria for authorship for this article, take responsibility for the integrity of the work as a whole, and have given their approval for this version to be published.

Author Contributions. Balaji Veeraraghavan conceived and designed the commentary with an important intellectual content. Yamuna Devi Bakthavatchalam drafted with manuscript and critically analyzed the literature data. Rani Diana Sahni critically revised the manuscript. All authors read and approved the final manuscript.

Disclosures. Balaji Veeraraghavan, Yamuna Devi Bakthavatchalam, and Rani Diana Sahni declare that they have no conflict of interest.

Compliance with Ethics Guidelines. This article is based on previously conducted studies and does not contain any studies with human participants or animals performed by any of the authors.

Data Availability. Data sharing is not applicable to this article as no datasets were generated or analyzed during the current study.

Open Access. This article is licensed under a Creative Commons Attribution-NonCommercial 4.0 International License, which permits any non-commercial use, sharing, adaptation, distribution and reproduction in any medium or format, as long as you give appropriate credit to the original author(s) and the source, provide a link to the Creative Commons licence, and indicate if changes were made. The images or other third party material in this article are included in the article's Creative Commons licence, unless indicated otherwise in a credit line to the material. If material is not included in the article's Creative Commons licence and your intended use is not permitted by statutory regulation or exceeds the permitted use, you will need to obtain permission directly from the copyright holder. To view a copy of this licence, visit http://creativecommons.org/licenses/by$\mathrm{nc} / 4.0 /$.

\section{REFERENCES}

1. Robilotti E, Deresinski S. Carbapenemase-producing Klebsiella pneumoniae. F1000Prime Rep. 2014;6:80.

2. D'Angelo RG, Johnson JK, Bork JT, et al. Treatment options for extended-spectrum beta-lactamase (ESBL) and AmpC-producing bacteria. Expert Opin Pharmacother. 2016;17(7):953-67.

3. van Duin D, Doi Y. The global epidemiology of carbapenemase-producing Enterobacteriaceae. Virulence. 2017;8(4):460-9.

4. Sanchez GV, Master RN, Karlowsky JA, et al. In vitro antimicrobial resistance of urinary Escherichia coli isolates among US outpatients from 2000 to 2010. Antimicrob Agents Chemother. 2012;56(4):2181-3.

5. Kaye KS, Gupta V, Mulgirigama A, et al. Antimicrobial resistance trends in urine Escherichia coli isolates from adult and adolescent females in the United States from 2011 to 2019: rising ESBL strains and impact on patient management. Clin Infect Dis. 2021; ciab560.

6. Hassuna NA, Khairalla AS, Farahat EM, et al. Molecular characterization of extended-spectrum $\beta$ lactamase-producing E. coli recovered from community-acquired urinary tract infections in Upper Egypt. Sci Rep. 2020;10(1):2772.

7. Zavala-Cerna MG, Segura-Cobos M, Gonzalez R, et al. The clinical significance of high antimicrobial resistance in community-acquired urinary tract infections. Can J Infect Dis Med Microbiol. 2020;2020:2967260.

8. Camacho-Cruz J, Martinez JM, Cufino JM, et al. Extended-spectrum b-lactamase-producing enterobacteriaceae causing community-acquired urinary tract infections in children in Colombia. Indian Pediatr. 2021;58(2):144-8.

9. Kresken M, Pfeifer Y, Hafner D, et al. Occurrence of multidrug resistance to oral antibiotics among Escherichia coli urine isolates from outpatient departments in Germany: extended-spectrum $\beta$ - 
lactamases and the role of fosfomycin. Int $\mathrm{J}$ Antimicrob Agents. 2014;44(4):295-300.

10. Alexandre K, Réveillon-Istin $\mathrm{M}$, Fabre R, et al. Temocillin against enterobacteriaceae isolates from community-acquired urinary tract infections: low rate of resistance and good accuracy of routine susceptibility testing methods. J Antimicrob Chemother. 2018;73(7):1848-53.

11. Cristea VC, Gheorghe I, Czobor Barbu I, et al. Snapshot of phylogenetic groups, virulence, and resistance markers in Escherichia coli uropathogenic strains isolated from outpatients with urinary tract infections in Bucharest, Romania. Biomed Res Int. 2019;2019:5712371.

12. van Driel AA, Notermans DW, Meima A, et al. Antibiotic resistance of Escherichia coli isolated from uncomplicated UTI in general practice patients over a 10-year period. Eur J Clin Microbiol Infect Dis. 2019;38(11):2151-8.

13. Findlay J, Gould VC, North P, et al. Characterization of cefotaxime-resistant urinary Escherichia coli from primary care in South-West England 2017-2018. J Antimicrob Chemother. 2020;75(1): $65-71$.

14. Larramendy S, Gaultier A, Fournier JP, et al. Local characteristics associated with higher prevalence of ESBL-producing Escherichia coli in community-acquired urinary tract infections: an observational, cross-sectional study. J Antimicrob Chemother. 2021;76(3):789-95.

15. Fasugba O, Mitchell BG, Mnatzaganian G, et al. Five-year antimicrobial resistance patterns of urinary Escherichia coli at an Australian tertiary hospital: time series analyses of prevalence data. PLoS ONE. 2016;11(10):e0164306.

16. Patwardhan V, Kumar D, Goel V, et al. Changing prevalence and antibiotic drug resistance pattern of pathogens seen in community-acquired pediatric urinary tract infections at a tertiary care hospital of North India. J Lab Physicians. 2017;9(4):264-8.

17. Gupta S, Kapur S, Padmavathi D. Comparative prevalence of antimicrobial resistance in community-acquired urinary tract infection cases from representative states of northern and southern India. J Clin Diagn Res. 2014;8(9):CD09-12.

18. Kumar N, Chatterjee $\mathrm{K}$, Deka $\mathrm{S}$, et al. Increased isolation of extended-spectrum beta-lactamaseproducing Escherichia coli from community-onset urinary tract infection cases in Uttarakhand, India. Cureus. 2021;13(3):e13837.

19. Zwane T, Shuping L, Perovic O. Etiology and antimicrobial susceptibility of pathogens associated with urinary tract infections among women attending antenatal care in four South African tertiary-level facilities, 2015-2019. Antibiotics (Basel). 2021;10(6):669.

20. Chervet D, Lortholary O, Zahar JR, et al. Antimicrobial resistance in community-acquired urinary tract infections in Paris in 2015. Med Mal Infect. 2018;48(3):188-92.

21. Mamani M, Nobari N, Alikhani MY, et al. Antibacterial susceptibility of Escherichia coli among outpatients with community-acquired urinary tract infection in Hamadan Iran. J Glob Antimicrob Resist. 2015;3(1):40-3.

22. Mantadakis E, Vouloumanou EK, Panopoulou M, et al. Susceptibility patterns of uropathogens identified in hospitalised children with community-acquired urinary tract infections in Thrace Greece. J Glob Antimicrob Resist. 2015;3(2):85-90.

23. Ayubi E, Safiri S. Worrisome high frequency of extended-spectrum beta-lactamase-producing Escherichia coli in community-acquired urinary tract infections: a case-control study; methodological issues. Int J Infect Dis. 2018;66:147-8.

24. Pérez Heras I, Sanchez-Gomez JC, Beneyto-Martin $P$, et al. Community-onset extended-spectrum $\beta$ lactamase producing Escherichia coli in urinary tract infections in children from 2015 to 2016: Prevalence, risk factors, and resistances. Medicine (Baltimore). 2017;96(50):e8571.

25. Priyadharshana U, Piyasiri LB, Wijesinghe C. Prevalence, antibiotic sensitivity pattern and genetic analysis of extended spectrum beta lactamase producing Escherichia coli and Klebsiella spp among patients with community acquired urinary tract infection in Galle district Sri Lanka. Ceylon Med J. 2019;64(4):140-5.

26. Kanda N, Hashimoto H, Sonoo T, et al. Gram-negative organisms from patients with community-acquired urinary tract infections and associated risk factors for antimicrobial resistance: a single-center retrospective observational study in Japan. Antibiotics (Basel). 2020;9(8):438.

27. Park JJ, Seo YB, Lee J. Antimicrobial susceptibilities of enterobacteriaceae in community-acquired urinary tract infections during a 5-year period: a single hospital study in Korea. Infect Chemother. 2017;49(3):184-93.

28. Kresken M, Körber-Irrgang B, Biedenbach DJ, et al. Comparative in vitro activity of oral antimicrobial agents against Enterobacteriaceae from patients with community-acquired urinary tract infections in three European countries. Clin Microbiol Infect. 2016;22(1):63.e1-63.e5. 
29. Stefaniuk E, Suchocka U, Bosacka K, et al. Etiology and antibiotic susceptibility of bacterial pathogens responsible for community-acquired urinary tract infections in Poland. Eur J Clin Microbiol Infect Dis. 2016;35(8):1363-9.

30. de Souza-da-Silva AP, de Sousa VS, de Araújo-Longo LG, et al. Prevalence of fluoroquinolone-resistant and broad-spectrum cephalosporin-resistant community-acquired urinary tract infections in Rio de Janeiro: impact of Escherichia coli genotypes ST69 and ST131. Infect Genet Evol. 2020;85:104452.

31. Critchley IA, Cotroneo N, Pucci MJ, et al. The burden of antimicrobial resistance among urinary tract isolates of Escherichia coli in the United States in 2017. PLoS ONE. 2019;14(12):e0220265.

32. Medina M, Castillo-Pino E. An introduction to the epidemiology and burden of urinary tract infections. Ther Adv Urol. 2019;11:1756287219832172.

33. Simmering JE, Tang F, Cavanaugh JE, et al. The increase in hospitalizations for urinary tract infections and the associated costs in the United States, 1998-2011. Open Forum Infect Dis. 2017;4(1):e281.

34. Schurek KN, Wiebe R, Karlowsky JA, et al. Faropenem: review of a new oral penem. Expert Rev Anti Infect Ther. 2007;5(2):185-98.

35. McLeod N, Carter M, Hackel R, et al. The novel $\beta$ lactamase inhibitor ETX1317 effectively restores the activity of cefpodoxime against extended spectrum $\beta$-lactamase (ESBL)- and carbapenemase-expressing enterobacteriaceae isolated from recent urinary tract infections. ASM Microbe, Atlanta, United States, 2018 Session 062.

36. Mcleod S Moussa S, Huband M, et al. The novel beta-lactamase inhibitor ETX1317 effectively restores the activity of cefpodoxime against recent global Enterobacteriaceae isolates from urinary tract infections. In: European Congress of Clinical Microbiology and Infectious Diseases, Amsterdam, the Netherlands, 2019 Abstract P1184.

37. Mendes ER, Rhomberg PR, Watters AA, et al. In vitro activity of the orally available ceftibuten/ VNRX-7145 combination against a challenge set of Enterobacteriaceae pathogens carrying molecularly characterised beta-lactamase genes. In: European Congress of Clinical Microbiology and Infectious Diseases, Amsterdam, the Netherlands, 2019 Abstract P1180.

38. Hackel M, Sahm D. In vitro activity of ceftibuten in combination with VNRX-7145 and COMPARATORS against 1066 UTI isolates non-susceptible to amoxicillin-clavulanate and levofloxacin. ASM Microbe 2019, San Francisco, CA, June 20-24, 2019.
39. Duncan LR, Rhomberg PR, Mendes RE, et al. Ceftibuten-avibactam activity against $\beta$-lactam-resistant Enterobacteriaceae clinical isolates. ASM Microbe San Francisco, California, 2019 Abstract AAR-717.

40. Castanheira M, Doyle LTB, Davis AP, et al. Activity of novel $\beta$-lactamase inhibitor QPX7728 combined with $\beta$-lactams against ST258 Klebsiella pneumoniae and ST131 Escherichia coli isolates producing $\beta$-lactamases. Open Forum Infect Dis. 2020;7(1):S212-3.

41. Critchley AI, Cotroneo NS, Pucci MJ, et al. Tebipenem: an oral carbapenem with activity against multi-drug resistant urinary tract infection isolates of Escherichia coli collected from US Medical Centers during 2019. Open Forum Infect Dis. 2020;7(1): S831.

42. Arends SJR, Rhomberg PR, Cotroneo N, et al. Antimicrobial activity evaluation of tebipenem (SPR859), an orally available carbapenem, against a global set of enterobacteriaceae isolates, including a challenge set of organisms. Antimicrob Agents Chemother. 2019;63(6):e02618-e2718.

43. Karlowsky JA, Adam HJ, Baxter MR, et al. In vitro activity of sulopenem, an oral penem, against urinary isolates of Escherichia coli. Antimicrob Agents Chemother. 2018;63(1):e01832-e1918.

44. Jain A, Utley L, Parr TR, et al. Tebipenem, the first oral carbapenem antibiotic. Expert Rev Anti Infect Ther. 2018;16(7):513-22.

45. Tebipenem. Spero Therapeutics licenses exclusive rights to SPR994, a novel oral carbapenem being studied for use in adults. 2017. https:// globenewswire.com/news-release/2017/10/10/11436 81/0/en/SperoTherapeutics-Licenses-Exclusive-Rights -to-SPR994-a-Novel-OralCarbapenem-Being-Studiedfor-Use-in-Adults.html. Accessed 26 Mar 2021.

46. Kato K, Shirasaka Y, Kuraoka E, et al. Intestinal absorption mechanism of tebipenem pivoxil, a novel oral carbapenem: involvement of human OATP family in apical membrane transport. Mol Pharm. 2010;7(5):1747-56.

47. Eckburg PB, Jain A, Walpole S, et al. Safety, pharmacokinetics, and food effect of tebipenem pivoxil hydrobromide after single and multiple ascending oral doses in healthy adult subjects. Antimicrob Agents Chemother. 2019;63(9):e00618-e619.

48. McEntee L, Johnson A, Farrington N, et al. Pharmacodynamics of tebipenem: new options for oral treatment of multidrug-resistant gram-negative infections. Antimicrob Agents Chemother. 2019;63(8):e00603-e619.

49. Lori AM, Susannah MW, Patricia AW, et al. Oral tebipenem pivoxil hydrobromide is non-inferior to 
IV ertapenem in complicated urinary tract infection (cUTI) and acute pyelonephritis (AP)—results from the pivotal ADAPT-PO study. Open Forum Infect Dis. 2020; 7:S844-5.

50. Minamimura $M$, Taniyama $Y$, Inoue $E$, et al. In vitro antibacterial activity and $\beta$-lactamase stability of CP-70,429 a new penem antibiotic. Antimicrob Agents Chemother. 1993;37:1547-51.

51. Sulopenem. Iterum announces sulopenem in development for treatment of Gram-negative, multidrug resistant infections. 2017. https://ir.iterumtx. $\mathrm{com} /$ press-releases/detail/5/iterum-announcessulopenem-in-development-for-treatment-of. Accessed 15 Apr 2021.

52. Dunne M, Dunzo E, Puttagunta S. A phase 1 study to assess the pharmacokinetics of sulopenem etzadroxil (PF-03709270). Open Forum Infect Dis. 2017;4:S525-6.

53. Sulopenem. Iterum therapeutics US. 2020. https:// www.sec.gov/Archives/edgar/data/1659323/000156 459021012694/itrm-10k_20201231.htm. Accessed 16 Apr 2021.

54. Michael WD, Anita FD, Michael Z, et al. Efficacy and safety of oral sulopenem etzadroxil/probenecid versus oral ciprofloxacin in the treatment of uncomplicated urinary tract infections (uUTI) in adult women: results from the SURE-1 trial. Open Forum Infect Dis. 2020;7:S844.

55. Michael WD, Steven IA. Efficacy and safety of intravenous sulopenem followed by oral sulopenem etzadroxil/probenecid versus intravenous ertapenem followed by oral ciprofloxacin or amoxicillinclavulanate in the treatment of complicated urinary tract infections (cUTI): results from the SURE-2 Trial. Open Forum Infect Dis. 2020;7:S636.

56. Butler MS, Paterson DL. Antibiotics in the clinical pipeline in October 2019. J Antibiot (Tokyo). 2020;73(6):329-64.

57. Veeraraghavan B, Bakthavatchalam YD, Sahni RD. Orally administered amoxicillin/clavulanate: current role in outpatient therapy. Infect Dis Ther. $2021 ; 10(1): 15-25$.

58. Stewart AG, Harris PNA, Henderson A, et al. Oral cephalosporin and $\beta$-lactamase inhibitor combinations for ESBL-producing Enterobacteriaceae urinary tract infections. J Antimicrob Chemother. 2020;75(9):2384-93.

59. Kakumanu VK, Arora V, Bansal AK. Investigation of factors responsible for low oral bioavailability of cefpodoxime proxetil. Int J Pharm. 2006;317(2): 155-60.
60. The United States Food and Drug Administration. Prescribing information for cefpodoxime proxetil. Sandoz GmbH. 2008. https://www.accessdata.fda. gov/drugsatfda_docs/label/2013/050674s015,0506 75s018lbl.pdf. Accessed 21 Apr 2021.

61. Durand-Réville TF, Comita-Prevoir J, Zhang J, et al. Discovery of an orally available diazabicyclooctane inhibitor (ETX0282) of class A, C, and D serine $\beta$ lactamases. J Med Chem. 2020;63(21):12511-25.

62. Miller AA, Shapiro AB, McLeod SM, et al. In vitro characterization of ETX1317, a broad-spectrum $\beta$ lactamase inhibitor that restores and enhances $\beta$ lactam activity against multi-drug-resistant enterobacteriales, including carbapenem-resistant strains. ACS Infect Dis. 2020;6(6):1389-97.

63. ETX0282. Entasis Therapeutics announces initial ETX0282 phase 1 results. Entasis Therapeutics Holdings Inc. 2019. https://www.globenewswire. com/news-release/2019/06/13/1868352/0/en/EntasisTherapeutics-Announces-Initial-ETX0282-Phase-1Results.html. Accessed 19 Apr 2021.

64. O'Donnell J, Tanudra A, Chen A, et al. Pharmacokinetic/pharmacodynamic determination and preclinical pharmacokinetics of the $\beta$-lactamase inhibitor ETX1317 and its orally available prodrug ETX0282. ACS Infect Dis. 2020;6(6):1378-88.

65. Radwanski E, Teal M, Affrime M, et al. Multipledose pharmacokinetics of ceftibuten in healthy adults and geriatric volunteers. Am J Ther. 1994;1: $42-8$.

66. Stein GE, Christensen S, Mummaw N. Treatment of acute uncomplicated urinary tract infection with ceftibuten. Infection. 1991;19:124-6.

67. Jodie H, Cassandra L, Chatwin P, et al. The orally bioavailable $\beta$-lactamase inhibitor VNRX-7145 restores bactericidal activity of ceftibuten against enterobacteriaceae expressing ambler class $\mathrm{A}, \mathrm{C}$ and D enzymes. ASM microbe. San Francisco, California, 2019 AAR-720.

68. Vázquez-Ucha JC, Arca-Suárez J, Bou G, et al. New carbapenemase inhibitors: clearing the way for the $\beta$-lactams. Int J Mol Sci. 2020;21(23):9308.

69. Avery LM, Abdelraouf K, Nicolau DP. In vivo pharmacodynamics of VNRX-7145 in the neutropenic murine thigh infection model when administered in combination with humanized exposures of twice daily ceftibuten (CTB) against serine $\beta$-lactamase-producing enterobacteriaceae (SBL-EB). Open Forum Infect Dis. 2019;6(Suppl 2): S311.

70. Merdjan H, Rangaraju M, Tarral A. Safety and pharmacokinetics of single and multiple ascending 
doses of avibactam alone and in combination with ceftazidime in healthy male volunteers: results of two randomized, placebo-controlled studies. Clin Drug Investig. 2015;35(5):307-17.

71. Gordon E, Duncton M, Lal R, et al. Oral prodrugs of avibactam, medicinal chemistry, and synthesis of ARX-1796. In: European Congress of Clinical Microbiology and Infectious Diseases, Amsterdam, the Netherlands, 2019 Abstract P1159.

72. ARX-1796. Arixa Pharmaceuticals announces acquisition by Pfizer's Hospital Business (press release). 2020. https://www.businesswire.com/news/ home/20201022005157/en/Arixa-PharmaceuticalsAnnounces-Acquisition-by-Pfizer\%E2\%80\%99sHospital-Business. Accessed 2 May 2021.

73. Trias J, Sable C, Hackel M, et al. Potentiation of oral cephalosporins and carbapenems by the addition of avibactam. In: European Congress of Clinical Microbiology and Infectious Diseases, Amsterdam, the Netherlands, 2019 Abstract P1151.

74. Sabet M, Tarazi Z, Griffith DC. In vivo activity of QPX7728, an ultrabroad-spectrum beta-lactamase inhibitor, in combination with beta-lactams against carbapenem-resistant Klebsiella pneumoniae. Antimicrob Agents Chemother. 2020;64(11): e01267-e1320.
75. Lomovskaya O, Tsivkovski R, Nelson K, et al. Spectrum of beta-lactamase inhibition by the cyclic boronate QPX7728, an ultrabroad-spectrum betalactamase inhibitor of serine and metallo-beta-lactamases: enhancement of activity of multiple antibiotics against isogenic strains expressing single beta-lactamases. Antimicrob Agents Chemother. 2020;64(6):e00212-e220.

76. Rubio-Aparicio D, Nelson K, Griffith CD, et al. In vitro activity in combination with oral beta-lactam antibiotics against enterobacteriaceae. ASM Microbe, San Francisco, California, 2019 Abstract AAR-710.

77. Hecker SJ, Reddy KR, Lomovskaya O, et al. Discovery of cyclic boronic acid QPX7728, an ultrabroadspectrum inhibitor of serine and metallo- $\beta$-lactamases. J Med Chem. 2020;63(14):7491-507.

78. Tarazi Z, Sabet M, Parkinson J, et al. Pharmacokinetics of QPX7728 following IV dosing in nonclinical species. ASM Microbe, San Francisco, California, 2019 Abstract AAR-713. 